\title{
Laboreal
}

Volume $8 \mathrm{~N}^{\circ} 2$ | 2012

A antropotecnologia, ferramenta ou engodo?

\section{Construcción de conocimientos y apropiación de nuevas técnicas de diagnóstico de fallas en operadores de talleres de reparación de automóviles}

Construção de conhecimentos e apropriação de novas técnicas de diagnóstico de avarias pelos operadores de oficinas automóveis

La construction de connaissances et l'appropriation de nouvelles techniques de diagnostic de pannes chez les opérateurs de " petits " garagistes

Knowledge construction and acquisition of new techniques for the diagnosis of failures by car repair operators

\section{Pablo Granovsky y Mário Poy}

\section{OpenEdition}

\section{Journals}

\section{Edición electrónica}

URL: http://journals.openedition.org/laboreal/6706

DOI: $10.4000 /$ laboreal. 6706

ISSN: 1646-5237

Editor

Universidade do Porto

\section{Referencia electrónica}

Pablo Granovsky y Mário Poy, « Construcción de conocimientos y apropiación de nuevas técnicas de diagnóstico de fallas en operadores de talleres de reparación de automóviles », Laboreal [En línea], Volume 8 N² | 2012, Publicado el 01 diciembre 2012, consultado el 11 octubre 2019. URL : http:// journals.openedition.org/laboreal/6706; DOI : 10.4000/laboreal.6706

Este documento fue generado automáticamente el 11 octubre 2019

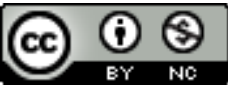

Laboreal está licenciado com uma Licença Creative Commons - Atribuição-NãoComercial 4.0 Internacional. 


\section{Construcción de conocimientos y apropiación de nuevas técnicas de diagnóstico de fallas en operadores de talleres de reparación de automóviles}

Construção de conhecimentos e apropriação de novas técnicas de diagnóstico de avarias pelos operadores de oficinas automóveis

La construction de connaissances et l'appropriation de nouvelles techniques de diagnostic de pannes chez les opérateurs de " petits " garagistes

Knowledge construction and acquisition of new techniques for the diagnosis of failures by car repair operators

Pablo Granovsky y Mário Poy

NOTA DEL EDITOR

Manuscrito recibido en : Agosto/2012

Aceptado tras peritaje : Noviembre/2012

\section{Introducción}

Los procesos de innovación tecnológica implican grandes desafíos conceptuales para las diferentes disciplinas -humanas y sociales- interesadas por el mundo del trabajo, cuenta habida de los impactos que dichos procesos conllevan : en un plano cultural, en cuanto a la diversidad de los contextos socioculturales donde esas tecnologías se transfieren e implantan (Wisner, 1981, 1985), en el plano cognitivo, a causa de las 
modificaciones que dichas innovaciones implican respecto de las transformaciones en el contenido de las tareas (Leplat, 2000), en el plano complementario de la actividad, en cuanto a la modificación o construcción de nuevos esquemas de utilización de los artefactos (Folcher \& Rabardel, 2009, Rabardel, 1995), en la transformación o la construcción de nuevos conocimientos mediante los objetos técnicos transferidos (Geslin, 2004), y en el de la formación a partir de la acción (Schön, 1998).

2 Guardando como telón de fondo estos cuatro ejes complementarios -el cultural, el cognitivo, el de la actividad instrumental y el de la reflexividad-, el presente artículo se propone describir y comprender el impacto del cambio tecnológico que significó la introducción de la electrónica a los componentes del automóvil, en el trabajo de los operadores de talleres mecánicos, teniendo en consideración los procesos de aprendizaje en la transición de la mecánica tradicional a la electrónica.

3 En particular, este estudio se centra en el trabajo de los operadores del Servicio Mantenimiento y de Reparación de Automóviles (SMyRA) de talleres independientes, organizaciones que están por fuera del circuito de los talleres de las concesionarias oficiales de autos, y por lo tanto no dependientes de los fabricantes.

4 Desde el enfoque de la antropotecnología, el caso abordado resulta pertinente ya que lleva a preguntarse por las formas de apropiación y de elaboración de conocimientos en aquellos actores de la cadena de valor automotriz [1] que están por fuera de los "márgenes del perímetro" de la transferencia tecnológica.

5 En efecto, el hecho de estar por fuera de las "islas antropotecnológicas" (Wisner, op. cit. p. 81), afecta directamente al trabajo de los talleres independientes. La identificación de los desperfectos en los autos se realiza mediante el uso de nuevos artefactos técnicos (osciloscopio, scanner, tester), con la ayuda de los manuales del fabricante (las terminales automotrices) que brindan exclusivamente a las concesionarias oficiales los parámetros para realizar el diagnóstico por cada marca y modelo de vehículo. La falta de acceso a esa información coloca a los talleres independientes en una situación particular que hace interesante su estudio.

6 Si bien uno de los pilares en los que se ha erigido la antropotecnología ha sido el de prevenir los riesgos para la salud y la seguridad de las poblacionales locales (en éste caso, los talleres independientes) hacia las cuales las "tecnologías viajan" en este caso, el de los talleres independientes, la problemática se desliza hacia una cuestión más esencial : no se trata ya del problema del impacto de la transferencia tecnológica sobre la salud y la seguridad sino de la simple supervivencia de los talleres mediante la apropiación de los nuevos objetos técnicos.

7 Tal como lo señala Geslin (op. cit. p. 76) la mayoría de los estudios realizados entre los años 70 y 90 sobre transferencia de tecnologías han sido de carácter más bien económico y han puesto el énfasis en las modalidades de implantación y alimentado la reflexión sobre las formas que deberían revestir para ser exitosas. En nuestro caso, el interés se centra en el análisis de las maneras en que, los conocimientos de los operadores a partir de la introducción de un objeto técnico, se construyen y se transforman a través de la acción (Geslin, Ibid.).

8 La elección, por lo tanto, de indagar sobre el trabajo de operadores de talleres independientes, obedece a una doble razón :

- Por un lado, a la posibilidad de poder estudiar -dentro de la cadena automotriz al "eslabón más débil" en términos de transferencia de tecnologías de equipos e información, de 
instalaciones y de respaldo financiero, las formas de apropiación de estas nuevas tecnologías y de construcción de nuevos conocimientos, en un contexto de adaptación a dichas innovaciones.

- Por otro lado, se trata de poner en evidencia las estrategias de ajuste desarrolladas por estos actores para la construcción de nuevas competencias (Rogalski \& Marquié, 2004), mediante la actividad de reflexión en la acción (Schön, op. cit.) en la búsqueda de fallas en los sistemas electrónicos de los automóviles, en situaciones de trabajo reales.

La reflexión en torno a un caso particular, el que nos ocupa, de lo que podría denominarse una "transferencia tecnológica desajustada" (Wisner, op. cit.), en tanto deja por fuera del proceso numerosa cantidad de actores, pretende realizar un aporte a la reflexión al campo de la antropotecnología en la medida que interroga acerca de los efectos que dicha transferencia produce respecto de las formas de apropiación y de uso de objetos técnicos, de las formas de actuar y de pensar de las personas en contextos diferentes de los que dichos objetos fueron diseñados (Geslin op. cit.). Y dicha reflexión cobra aún más relevancia en la medida que de la incorporación exitosa de estos dispositivos técnicos, depende la supervivencia y la permanencia de dichos actores dentro del tejido socio productivo, en este caso, los talleres independientes de reparación de autos.

El presente trabajo, parte de una serie de investigaciones dentro del sector automotriz[1], se inscribe en el marco en que los actores del campo empresario, del sindical, así como los de las instituciones de formación vinculadas a la mecánica del automotor, se encuentran reformulando sus estrategias formativas orientadas al segmento de mecánicos formados en la mecánica "tradicional", objetos en este estudio, y en la "reconversión" de sus competencias a partir de la introducción de la electrónica en los componentes del automóvil. Por ello, este trabajo brindó elementos y colaboró en la actualización y "modernización" de currículas formativas, materiales didácticos, y en la fundamentación de requerimientos de actualización en equipos de diagnóstico para las instituciones formativas. En particular, este estudio se articula en torno al análisis de dos casos reales, en dos talleres, y sobre dos problemas de diagnóstico diferentes intentando guardar toda la riqueza de las situaciones en los que se refiere las diferentes interacciones (los diálogos entre operadores, entre operadores y cliente, entre colegas y las interacciones con los diferentes artefactos técnicos).

\section{Evoluciones y determinantes tecnológicos en el SMyRA en el contexto argentino}

11 El parque automotor en Argentina constituido durante décadas por modelos diseñados en los años 60 por las casas matrices - Ford Falcon, Renault 12, Peugeot 504, Fiat 147 -, fue renovado y modernizado a partir de la década del ' 90 por vehículos muy cercanos a la frontera tecnológica (Delfini, Dubbini, \& Rivero, 2007). Al mismo tiempo, el mercado del automóvil comenzó a caracterizarse por la diversidad de marcas y modelos nacionales e importados - existentes.

Las nuevas unidades van a expresar un cambio tecnológico fundamental: el funcionamiento del automóvil ya no se regirá por sistemas mecánicos relativamente autónomos, sino por sistemas electrónicos cuyos componentes se integran funcionalmente por la acción de microprocesadores. 
13 La tecnología electrónica comenzó reemplazando los sistemas convencionales de encendido y alimentación de combustible - sistemas de encendido electrónico e inyección electrónica -, y luego se incorporó en el resto de los sistemas del automóvil : Sistema de Frenos ABS, Transmisiones, Sistemas de Seguridad, entre otros. La incorporación de estas tecnologías exigió nuevas inversiones en instrumental y complejizó las actividades vinculadas al diagnóstico y reparación de fallas en estos sistemas (Leplat, op. cit.). Estas transformaciones, impactaron a su vez en la estructura ocupacional del sector reestructurando, eliminando y definiendo nuevos roles profesionales (Granovsky, 2010).

La composición interna de este sector laboral se destaca por presentar dos tipos de estructuras empresariales claramente diferenciadas: los talleres de las concesionarias oficiales de autos; los talleres independientes.

15 Ambas estructuras presentan claras diferencias en aspectos tales como, entre otras: inserción en el mercado, política de inversiones, relaciones con las terminales automotrices, tecnología. En un plano más concreto, mientras que las primeras se benefician de una "transferencia tecnológica integral" (Wisner, op. cit.), es decir no solamente los artefactos técnicos (scanners, manuales de procedimientos de detección de fallas), sino también de la provisión de las herramientas pedagógicas necesarias para la transformación de las competencias de los operadores, las segundas apelan a diferentes estrategias de apropiación, que más adelante detallamos, que permitan paliar esta situación.

16 Para el diagnóstico de fallas en los sistemas electrónicos, el operador debe poseer el manual del automóvil, de acuerdo a su marca y modelo, que brinda los parámetros para poder identificar las fallas, estableciendo el rango de los valores de funcionamiento normal de los distintos componentes. Pero, por política comercial de las empresas terminales (fabricante del automóvil), dicho manual sólo es distribuido a su cadena comercial, es decir las concesionarias oficiales. De esta forma, los talleres independientes tienen grandes dificultades para diagnosticar fallas, sobre todo en los modelos de automóvil más modernos, razón que los obliga a incursionar en un circuito informal de circulación de dicha información.

\section{2. ¿Qué artefactos y cómo se los incorpora en los talleres independientes?}

17 La incorporación de tecnología en los talleres, se concentra en tres artefactos centrales : el téster, el scanner y el osciloscopio.

18 El téster permite diagnosticar las instalaciones eléctricas e identificar posibles fallas cortocircuitos- en las mismas. Existen tésters que son especiales para inyección y que permiten también medir parámetros auxiliares que son útiles, como ser el tiempo de inyección, las RPM, las frecuencias. Este tipo de objeto técnico es el de mayor difusión, el más económico, y el de menor complejidad de uso.

19 El scanner es el artefacto central para trabajar con los dispositivos electrónicos del automóvil. Permite identificar fallas en los distintos sistemas electrónicos. "Interpreta", de acuerdo al software que posee (manual del automóvil de acuerdo a marca y modelo), a partir del diálogo que mantiene con la unidad de control del auto (computadora central del automóvil que gestiona sus dispositivos electrónicos), la 
información que recibe de los sensores - dispositivos que brindan información a la unidad de control del auto sobre posibles fallas y que están presentes en los distintos componentes que conforman los sistemas del automóvil- permitiendo identificar que el problema se encuentra en un determinado sistema o conjunto de componentes.

Es necesario aclarar que las terminales automotrices transfieren a sus concesionarias oficiales scanners de la marca específica con mayor capacidad para diagnosticar fallas en dichos modelos, mientras que los talleres independientes deben recurrir a proveedores de equipos "multimarca" que requieren, para cada intervención, una adecuación a la marca y modelo específica con las dificultades correspondientes.

21 El osciloscopio que es una herramienta que mide en tiempo real, de manera gráfica, todas las señales de corriente en forma de onda en una frecuencia determinada. Trabaja en formato digital y permite identificar fallas cuando el scanner no lo permite, al ver el comportamiento del componente. Este tipo de fallas son aquellas que no se presentan con regularidad, sino que ocurren aleatoriamente.

El uso de estas herramientas por parte de los operadores para diagnosticar fallas en los sistemas electrónicos, permiten caracterizar tres modalidades -no excluyentes- de adquisición y apropiación de estos objetos técnicos, en los talleres independientes.

- En primer lugar, mediante capacitaciones informales, dictadas generalmente por mecánicos de concesionarias oficiales -que trabajan con estas tecnologías en sus modelos más avanzados- y que en acuerdo con mecánicos independientes explican y desarrollan en cursos breves, el funcionamiento básico del objeto técnico.

- En segundo lugar, los proveedores de tecnología, que en la mayoría de los casos comercializan equipos "multimarca", transfieren las formas de uso de los artefactos, como parte de la comercialización de dicha tecnología.

- Por último, y en el contexto específico del taller, mediante distintas formas de "experimentación”, de pruebas con fallas reales, los mecánicos de talleres independientes logran "dominar" estas tecnologías, y aquellos con mayor experiencia colaboran en la formación del resto de los operadores. El dominio de la tecnología esta centralmente vinculado a los programas para identificar fallas y el proceso de transferencia se relaciona con el uso de dichos programas y su adaptación a cada marca y modelo.

En este sentido, este estudio hace foco sobre esta última modalidad a fin de caracterizar los mecanismos por los cuales los mecánicos se apropian del uso de estos artefactos, luego de incorporar los conocimientos sistematizados en cursos, manuales, a sus prácticas cotidianas en el diagnóstico de fallas vinculadas a los sistemas electrónicos del automóvil.

\section{Los casos estudiados: características y modalidades de abordaje}

Para la realización de este estudio, y dadas las características de nuestra problemática, se seleccionaron 2 talleres con instrumental para el diagnóstico de sistemas electrónicos y que trabajen con modelos de vehículos con dispositivos electrónicos, privilegiando la indagación mediante estudios intensivos de casos.

Se buscó poder reconstruir mediante un tipo de estudio cualitativo las experiencias vinculadas con los cambios asociados a la introducción de la electrónica en los componentes del automóvil, a partir de la actividad de los propios operadores que 
debieron reconvertir sus competencias de la mecánica tradicional a la electrónica. Esto implicó un trabajo de campo de aproximadamente 1 año de reiteradas visitas a los talleres mecánicos referidos en los "casos".

La técnica escogida para el relevamiento de los datos fue la observación de los casos, acompañadas de verbalizaciones consecutivas de lo realizado, con la intención de reconstruir las prácticas de diagnóstico de los mecánicos, las relaciones de estas prácticas con las innovaciones en los talleres independientes en cuanto a gestión y tecnología.

Para la definición del universo de trabajo, se retuvo aquellos operadores que vivieron el proceso de conversión de la mecánica a la electrónica, es decir con un mínimo de 10 años de antigüedad en el sector y principalmente con experiencia en los sistemas de alimentación tradicionales y electrónicos.

Para poder abordar los casos a través de dos ejemplos reales tratados por mecánicos, se reconstruyeron tanto las secuencias de indagación, como las modalidades de intervención en la búsqueda de fallas en los sistemas electrónicos del automóvil.

El concepto de reflexión en la acción desarrollado por Schön (op. cit.) que refiere a los procesos reflexivos que tienen lugar en simultáneo al ejercicio de la actividad y se desarrollan "desde" la actividad misma, resulta una adecuada malla de análisis dada la presencia cada vez más significativa de instancias de reflexión en los contextos laborales contemporáneos y porque permite pensar, en el caso de los mecánicos, el desarrollo de competencias para el diagnóstico de fallas en los sistemas electrónicos del automóvil, como un proceso donde la reflexión desde y para la acción es un elemento central.

30 Se procedió a relevar las verbalizaciones de 5 mecánicos formados en las dos tecnologías (mecánica y electrónica) pidiéndoles un relato pormenorizado de los casos seleccionados, tanto en el momento de las intervenciones sobre de las fallas, como con posterioridad a su tratamiento. Lo que se detalla a continuación son dos casos de diagnóstico de fallas. En el primero, se describe toda la secuencia de diagnóstico a través de las verbalizaciones, complementadas en este caso por observaciones de la actividad de los operadores, durante las distintas instancias de búsqueda del origen de la falla (Guèrin, Laville, Daniellou, Duraffourg \& Kerguelen, 2006). En el segundo, se trata de la reconstrucción de un caso a través del relato de los mecánicos participantes y en donde se puede re-trazar la secuencia de indagación, hasta identificar el origen de la falla.

\section{El caso del auto "que no arrancaba" : estructuración del problema y experimentación}

\section{a) La estructuración del problema}

31 En el contexto del taller mecánico se puede observar cómo reiterados procesos de búsqueda de fallas (Rouse \& Rouse 1979) en los sistemas electrónicos del automóvil y la interacción entre saberes tácitos (Leplat, 1997) y codificados (Nonaka \& Takeuchi, 2002) en dichos contextos, son la base para el desarrollo de competencias de diagnóstico por parte de los operadores formados en la mecánica tradicional. 

un auto de gama baja (Volkswagen Gol modelo 1997), y donde el cliente manifiesta la presencia de recurrentes problemas con el "arranque" de su automóvil. En este sentido los operadores plantean el problema práctico como "un caso único", atendiendo a sus peculiaridades, buscando las características concretas de la situación, a partir de un descubrimiento gradual de distintas posibilidades de fallas. En ningún caso el problema está dado, sino que se trata de dar un nuevo sentido a la situación problemática. En esta determinación del problema los mecánicos manejan en forma selectiva grandes cantidades de información, deben mantener varios modos de considerar las cosas (el origen de la falla en el arranque puede estar en la correa de distribución, en el sensor de mariposa, en el tanque de nafta, en el cableado, etc.) sin interrumpir el flujo de la indagación en curso.

rmulan el problema, sugieren una dirección para reorganizar la situación y dirigen un conjunto de "experimentos" (test de carretera, indagación con el cliente, manipulación de distintos componentes, uso del scanner, etc.) tendientes a descubrir qué consecuencias e implicaciones pueden hacerse a partir de los mismos. Tratan de adaptar la situación al marco, a través de una red de acciones, consecuencias descubiertas, implicaciones, apreciaciones y acciones posteriores.

Dentro de esta red de acciones y reacciones se provocan fenómenos que han de ser comprendidos, problemas que han de ser resueltos $u$ oportunidades que han de explotarse. Los operadores desarrollan experimentos locales contenidos dentro de otros mayores (manipulación de componentes en el marco del proceso de diagnóstico con el scanner). Las acciones también producen cambios inintencionados que proporcionan nuevos significados a las situaciones. La situación "replica", los mecánicos "escuchan" y al apreciar lo que están oyendo reestructuran la situación una vez más.

En este caso particular, en que el auto no arrancaba, los mecánicos suponen que es la correa de distribución que se cortó, por lo que la cambian, pero el auto sigue "tironeando".

\footnotetext{
“...No arrancaba (el vehículo) y el primer síntoma que hizo fue que cortó la correa de distribución, ese fue el problema, no arranca porque es la correa, ya está dijimos : la correa arrancó pero el auto andaba como un caballo. Él (cliente) decía esto: "yo paso sobre una calle empedrada", decía: "me tironea, me tironea y se para" y le digo : "bueno, bueno... que se yo, debe ser esto...", lo scanneamos (uso del artefacto para identificar la falla), probamos, cambiamos algunas cosas, les pusimos y le sacamos otras (manipulación de componentes)..." (Gustavo)
}

Los operadores (el oficial y su ayudante) abordan la situación con una estructuración del problema gracias a la cual "sienten" que pueden resolverlo. No saben cual será la solución, pero el marco que imponen a la situación los lleva a un método de indagación en el que tienen confianza. Las acciones de los operadores (cambiar la correa de distribución) producen efectos no pretendidos y evalúan su experimento de determinación del problema estableciendo si les "gustan" estos cambios no intencionados o si les "gusta" lo que pueden hacer con ellos. Los mecánicos evalúan de acuerdo a su percepción de las potencialidades de coherencia y congruencia de las que se pueden dar cuenta a través de su nueva indagación. 
37 Los mecánicos evalúan su reestructuración de la situación problemática por su potencialidad para resolver el problema (identificar la falla), por sus apreciaciones sobre los efectos involuntarios de la acción, y también evalúan en función de mantener la investigación en marcha (puede ser la correa, el sensor de mariposa, el tanque de nafta, el cableado, etc.).

Como segundo paso lo "scanean" y el resultado les revela cuatro posibles fallas que corresponden a cuatro componentes distintos, le preguntan al cliente qué tipo de tironeo hace el vehículo, el tipo de ruido y comienzan a pensar cuáles pueden ser las posibles causas del tironeo.

“...lo scanneaste, empezás a ver que este no figura, esto no está relacionado con esto, lo sacamos, empezás a chequear, chequear, cuando ves que chequeaste un montón de cosas y de pronto sigue, lo que hizo el scanner capaz que tiene razón, entonces le cambias eso..." “...Cuando vos lo chequeas con el scanner y fijas el código de fallas, cuando el cliente me dice : "Y... me tironea" bueno... a mi me da estos componentes que me están figurando acá (en el scanner), esto es lo que me está haciendo esta falla, vos lo ponés en marcha y lo ves en tiempo real a ver qué componente, obviamente, lo mirás en tiempo real y funciona todo a la perfección, entonces intentás decir... "Bueno, si me tironea... ¿cómo me tironea ?, "chucu, chucu, chucu” ¿Qué es lo que me hace el "chucu, chucu, chucu" ?..." (Gustavo)

Los operadores no subsumen el problema bajo una categoría estándar, sino que la tratan como una entidad única, para la que deben inventar una descripción especialmente apropiada. Aunque el scanner indique que la falla se ubica en determinados componentes, ellos realizan "experimentos locales" con cada uno de los componentes para verificar lo que indica el scanner. Buscan abrir una línea de investigación explorando mediante heurísticos qué es lo diferente en esta experiencia única, ponen en movimiento una indagación sobre los rasgos peculiares del automóvil, escuchan al cliente, creando un conjunto particular de problemas. No aplican, en forma lineal, una regla extraída de la experiencia pasada, ni inventan algo totalmente nuevo.

“...entonces empezás a hacer como un proceso de lectura, decís : "Bueno, la manguera no me tironearía porque me aumentaría el consumo. El sensor de mariposa, que es el aparato que le dice a la computadora la presión, tampoco... porque si yo lo llevo a la misma posición y el auto pasa un pozo, se pararía enseguida". Empezás y descubrís tal "pirulito", entonces la máquina va a andar, ah!, joya! borrás la memoria y ya terminamos. Después resulta que el auto largaba charcos de nafta (al aplicar el test de carretera)... claro... anda mejor pero el defecto lo sigue haciendo. Pero ¿cuánto mejor ? Vuelvo a fijarme todos los defectos..." (Andrade).

El encuadre de la situación que hacen los operadores se estructura a partir de estos elementos, siguiendo una secuencia de descarte a partir de su experiencia que les indica "qué descartar" de acuerdo a las alternativas que indica el scanner y de las formas de experimentación que desarrollan (con el scanner, conversando con el cliente, manipulando componentes, etc.). Es su experiencia que le indica la secuencia de descarte, donde se manifiesta lo distintivo del mecánico experto, quién pone en juego un conjunto de guías tácitas construidas a lo largo de su historia laboral, para poder elegir entre diversos recorridos posibles en términos de la identificación de la falla. En el caso de que la falla se encuentre en la manguera de nafta la descartan porque en caso de falla el consumo de nafta aumentaría, y el cliente había negado este problema. 
41 En el caso del sensor de mariposa, que es el que le indica a la computadora la presión, lo descartan porque si la falla estuviese ahí, cuando el vehículo pasa por un "pozo" se pararía inmediatamente. Al consultar al cliente esto no pasaba.

Los operadores han desarrollado una guía tácita de indagación, de esquemas de utilización, de modos de intervención, de los ejemplos de otras marcas y modelos de vehículos, de los relatos escuchados de otros mecánicos, de las intervenciones, de las soluciones, de tipos de fallas de distintos automóviles de acuerdo a su marca y modelo. Los mecánicos ven la situación única como algo ya presente en su repertorio, sin poder verbalizar las similitudes y diferencias entre el ejemplar del repertorio (fallas típicas en un modelo y marca de automóvil) y la situación única. No la subsumen linealmente bajo una categoría o una regla. Desarrollan la capacidad para ver y hacer "como" ("como" si fuese un caso anterior similar en la marca y el modelo de automóvil y por consiguiente en el tipo de falla), lo que les permite tener el "tacto" necesario para afrontar los problemas que no se adecuan a las fallas que ya tiene "tipificadas", es decir ver situaciones desconocidas como familiares.

43 Es la amplitud y variedad del repertorio lo que brinda la capacidad de ver las situaciones desconocidas como elementos de su repertorio, por lo que no necesitan reducirlas a ejemplos de categorías estándar. Cada nueva experiencia de reflexión desde la acción enriquece su repertorio y, de esta forma, mediante los distintos casos es que se desarrollan estas competencias de diagnóstico.

44 La reflexión desde la acción en un caso único puede generalizarse a otros casos, no elevándola a la condición de principio general, sino mediante la contribución al repertorio del mecánico de los temas ejemplares a partir de los cuales, en los siguientes casos de su práctica, podrá desarrollar nuevas variaciones.

\section{b) La experimentación}

Los mecánicos, en su conversación reflexiva con una situación, a la que tratan como única e incierta, funcionan como un intermediario/experimentador. A través de su interacción con la situación-problema, le dan forma y se hacen parte de la misma.

La postura de los operadores transparenta una doble estrategia. Por un lado, deben conformar la situación de acuerdo a su propio encuadre pero, por otro, deben mantenerse abiertos ante "la réplica de la situación".

47 El encuadre del problema que establecen ante las alternativas de fallas en el caso del auto que no arranca, se vincula con la identificación de las siguientes líneas de indagación : la manguera de nafta, el sensor de mariposa, el tanque de nafta y el cableado

Como ya se dijo, la alternativa de que la falla se encuentre en la manguera de nafta la descartan porque el consumo de nafta aumentaría y el cliente había negado este problema. En el caso del sensor de mariposa, también lo descartan porque si la falla estuviese en el mismo, cuando el vehículo pasa por un "pozo" se pararía el auto inmediatamente. Al consultar al cliente esto no pasaba. De todas formas, controlan las conexiones de la manguera y el estado del sensor de mariposa (experimentos sobre la marcha).

49 Además al hacer un test de carretera el automóvil perdía nafta, entonces verifican el estado del tanque de nafta y era el correcto. Luego verifican electrónicamente (scanean) el sistema, para ver que sucedía con otros sensores y, como habían verificado 
varios componentes, evalúan el cableado del ECU (unidad de control del motor del auto) e identifican que algunos cables estaban sueltos y otros mal colocados, por lo que el problema era la instalación de cables del vehículo. El problema no eran los sensores sino que la información que enviaban los sensores a la computadora madre no llegaba adecuadamente.

“...entonces nos daban tres posibles componentes (sensores), lo que pasa es que antes de cambiar esos tres, chequeamos los cables que conectaban todo a la computadora..."'... Suponete que el sensor que nosotros le cambiamos... esté acá... esta información va a la computadora con un manojo de cables, el $90 \%$ de los casos los cables están bien porque nadie los toca, obviamente cuando yo abrí la instalación del auto, había cables sueltos, como yo le dije : "che el problema es de la instalación, y bueno... después descubrimos que había un problema con la instalación, le hicimos un montón de instalación nueva al auto..."

“...en realidad no era problema de los sensores, sino era de la información que no llegaba a la computadora..."'... Y fue ahí el tema, eran las conexiones de esos sensores..."

“... yo me acuerdo que cuando me lo trajo, por segunda vez, le digo: "che, mira, estoy haciendo algo mal en el auto”, “... yo con este auto tendría que cambiar todo (el cableado)", entonces... le vamos a cambiar todo"

"... había una ficha, que conectaba una cosa del sensor, que esa ya estaba rota, ellos la pegaron con Poxipol, una maderita, una artesanía bien hecha pero yo les dije "Mira, esto así no puede ser, vamos a poner las cosas bien (el cableado) sino nos vamos a volver locos"... chequeamos todas las instalaciones y algunas partes de las instalaciones también se las mejoré..." (Gustavo).

50 De esta forma, para llegar a identificar la falla en el cableado, además de la estructuración del problema, los mecánicos han desarrollado un proceso de "experimentación". Estos "dialogan" con sus situaciones que son experimentos de "reencuadre". Dentro del experimento más amplio de determinación de un problema, hay también experimentos locales variados. En esta relación del mecánico con las situaciones de falla, el artefacto le indica el área donde "tentativamente" estaría la falla, pero el diagnóstico en sí mismo, se relaciona con las acciones posteriores a esta identificación del "área", a partir de la secuencia de experimentos de reencuadre y del diálogo que establece el mecánico con los materiales de la situación.

51 El contexto práctico es diferente del contexto de investigación en varios modos importantes, que alude a cambiar las cosas y comprenderlas. El mecánico tiene interés en transformar la situación desde lo que es, hacia algo que le gusta más. También tiene interés en comprender la situación, pero al servicio de su interés en el cambio.

Los mecánicos realizan el experimento de prueba de hipótesis como un juego con la situación, buscando que esta se conforme a su hipótesis, pero permanecen abiertos a la posibilidad de que no sea así. La relación de los mecánicos con esta situación es transaccional. Dan forma a la situación, pero en su conversación con ella, para que sus propios modelos y apreciaciones sean también conformados por la situación. El interés de los mecánicos en cambiar la situación tiene prioridad sobre el interés de comprenderla. Este enfoque transaccional se relaciona con la "gestión" de la tensión entre buscar dar un marco a la situación en que se presenta la falla, manteniendo a la vez las distintas líneas de indagación abiertas hasta identificar el origen del problema. 
53 En síntesis, ante el primer síntoma cambian la correa como un experimento exploratorio que les permite seguir la indagación en curso, pero como el "tironeo" continúa experimentan con el scanner, con el test de carretera, escuchando al cliente y mediante la manipulación de cables y componentes. Estas formas de experimentación combinan el experimento exploratorio sobre la marcha, y la comprobación de hipótesis, priorizando en cada caso la coherencia del encuadre del problema, pero siempre manteniendo abiertas las varias posibilidades de resolución (la manguera de nafta, el sensor de mariposa, el tanque de nafta o el cableado).

54 En este primer caso, se puede comprobar cómo los mecánicos desarrollan conocimientos para diagnosticar fallas en los sistemas electrónicos a partir de integrar conocimientos de distintas bases tecnológicas, de la electrónica a la mecánica, de la informática a la electricidad. Esta integración, implica traducciones, decodificaciones y recodificaciones, y tiene lugar a partir del desarrollo de un enfoque "sistémico" vinculado a la incorporación de dispositivos electrónicos en el automóvil, útiles para la gestión de los distintos sistemas, incrementa las relaciones funcionales entre estos últimos (sistemas de alimentación, de frenos, de inyección, de transmisiones, etc.). En correspondencia con este incremento, la perspectiva sistémica -pensar en términos de los sistemas del automóvil y sus relaciones funcionales- les permite integrar las distintas bases tecnológicas. Estos elementos les otorgan una "ventaja" a los mecánicos con más experiencia, respecto de sus ayudantes. Estos últimos, incorporan esta perspectiva sistémica a partir de compartir experiencias comunes de resolución de problemas en los vehículos junto a los más experimentados, tal como queda expresado por uno de los mecánicos, respecto del uso del scanner para diagnosticar.

“...hoy un auto hace una falla, la hace hoy, quince días no las hace, después lo hace tres días seguidos, después no la vuelve a hacer, después un día no arranca más, entonces ponés el scanner que es tu equipo de diagnóstico que te permite dialogar con las unidades de control que tiene el vehículo, que son varias, por ejemplo un Mondeo 16 tiene ABS, airbag, climatización, el inflado de ruedas, aparcamiento, toda esa tecnología. Entonces, cuando vos tenés un problema y entrás con el scanner, el scanner no es una herramienta que te va a decir el problema esta acá, cambia este componente, sino que el scanner interpreta de acuerdo al software que tiene y al diálogo que hace con la unidad de control y de la información que recibe de los sensores y de lo que está pasando recibe una información que procesa, analiza y determina que el problema va para determinado lado, por ejemplo, es habitual encontrar que cuando vos pones el scanner y el vehículo a inyección tira humo negro te va a salir sonda de oxígeno defectuosa, ¿Por qué ? Porque está midiendo por valores muy altos del monóxido de carbono porque la sonda está midiendo mucha cantidad de nafta en exceso. Para darte un ejemplo mirá lo que pasó hace poco tiempo con uno nuevo (se refiere a un mecánico ayudante). "el scanner le decía sonda de oxigeno mal, fue y compró una sonda de oxígeno y la cambió ; y volvió a tener el mismo problema porque el auto seguía tirando humo negro y le salía de vuelta sonda de oxígeno mal, ¿Por qué ?, ¿Qué pasaba? La sonda de oxígeno no es que estaba mal, estaba corrigiendo en un valor límite, entonces al corregir en un valor límite, la unidad de control lo tomaba como un código de falla, y eso puede ser porque están sucios los inyectores y está tirando mucha nafta, porque tiene mucha presión de combustible. $Y$ todo esto te lo da la experiencia y sin esto no lo resolvés, con saber usar el scanner no te alcanza, tenés que conocer todos los sistemas y como se integra con la unidad de control..." (Juan). 

La segunda cuestión se relaciona con el desarrollo de distintas formas de “experimentación" en el proceso de diagnóstico. A partir de este caso, los mecánicos desarrollan un esquema transaccional con los "materiales" de cada situación problemática. Mediante el uso de los instrumentos de diagnóstico, los mecánicos desarrollan esquemas de indagación e intervención que les permiten ir "encuadrando" el proceso de diagnóstico. De esta forma, ellos "gestionan" la tensión entre buscar darle un marco a la situación de falla, y su repertorio, manteniendo las distintas líneas de indagación abiertas hasta identificar el origen de la falla. De esta manera, el proceso de apropiación de los nuevos artefactos tecnológicos se vincula a estas formas de experimentación y de aplicación de este esquema transaccional mediante la reflexión en la acción y la construcción de nuevos conocimientos

\section{El caso del auto al que le sale "humo negro" : racionalidad técnica versus reflexión en la acción}

57 Pensar el desarrollo de competencias de diagnóstico de fallas, a partir de la interacción entre conocimientos prácticos y codificados, requiere de una instancia destacada de reflexión en el contexto de los talleres mecánicos. Este concepto de reflexión se diferencia de la perspectiva de la "racionalidad técnica" (Schön, op. cit). Esta, parte de concebir la resolución de problemas instrumentales a través de la aplicación de la teoría científica y la técnica, desde un ajuste instrumental medios-fines. La secuencia "lineal" es que la aplicación de la ciencia básica produce ciencia aplicada, y esta brinda técnicas de diagnóstico y de solución de problemas. De acuerdo a esta perspectiva, sería posible seleccionar los medios apropiados a los fines correspondientes mediante la aplicación de la teoría científica apropiada fundando una "epistemología positivista de la práctica".

58 Desde este modelo, actividad profesional consiste en la resolución de problemas instrumentales que se han hecho rigurosos por la aplicación de la teoría científica y de la técnica. Solamente los profesionales, en el ajuste instrumental de los medios a los fines, practican rigurosamente la resolución técnica de los problemas, basándose en un conocimiento científico especializado.

Pero, las limitaciones de este modelo se relacionan con que en el mismo no "encajan" la complejidad, la incertidumbre, la variabilidad, el carácter particular de las situaciones de trabajo, tal como no ha cesado de remarcarlo desde hace mucho tiempo la ergonomía de la actividad (Wisner, op. cit. ; Guerin, Laville, Daniellou \& alt, op. cit).

60 Por el contrario en el enfoque de la "reflexión desde y para la acción" se manifiesta en la necesidad del "encuadre" donde los problemas no se presentan como dados, sino que deben ser construidos a partir de las interacciones vía los instrumentos permitiendo, al mismo tiempo, conocer las propiedades del objeto/problema -"mediaciones epistémicas"- y resolver los problemas que el mismo plantea "mediaciones pragmáticas" (Rabardel, op. cit.; Folcher \& Rabardel, op. cit.). Se busca así poder dar 
sentido a la situación, seleccionando a partir de qué "asuntos" tratar la situación, imponiendo coherencia a la misma, identificando en qué dirección modificar la situación, al mismo tiempo que enriqueciendo los propios esquemas de utilización.

61 El enfoque reflexivo puede ser caracterizado, en el caso de los mecánicos, a partir del siguiente ejemplo que refiere a un auto con inyección electrónica diesel al que "le sale humo negro". En este contexto lo primero que piensa el mecánico es qué significa, qué está implicando la salida de humo negro, concluyendo que hay una alteración en la mezcla de oxígeno y combustible : una mayor cantidad de combustible por cantidad de oxígeno.

“...un ejemplo de una falla convencional y esa misma falla en algo electrónico, el caso de un auto con motor diesel que larga humo negro y eso tiene que ver con la mezcla de combustible y oxígeno..." (Héctor).

Desde el enfoque de la reflexión desde la acción el conocimiento está implícito en nuestros patrones de acción y en nuestra sensación respecto de las cosas con las que estamos tratando. El conocimiento se da desde la acción, el mecánico siempre depende de reconocimientos, juicios y habilidades que son tácitos, es decir el saber se da desde la acción y no está asociado a una "operación intelectual previa".

Esto se manifiesta al pensar en "hacer algo" mientras se lo "está haciendo". Se reflexiona sobre la acción (el objeto de la reflexión es el propio proceso de trabajo), desde la acción (proceso de trabajo). Hay variación, combinación y recombinación de un conjunto de figuras dentro de un esquema que vincula y da coherencia a la actuación.

El operador intenta identificar qué sistemas del automóvil pueden estar relacionados con la salida de humo negro, a qué sistemas afecta. Para comenzar a realizar el diagnóstico tienen presente el sistema convencional del auto y el sistema electrónico (los sensores del auto principalmente) a partir de un principio ordenador del sentido de la indagación que es ir "de lo más fácil a lo más complejo".

Su repertorio le permite focalizarse en el control de tres cuestiones principales : el sistema de control de aire, el sistema de alimentación y el sensor de aire y la computadora del vehículo:
“...probás la entrada de aire, puede ser un filtro de aire tapado, después el combustible, ahí tenés que ver todo el sistema de alimentación, el tipo de gasoil, la bomba inyectora, la bomba alimentadora..."
“...el humo negro que va a tirar es por el filtro de aire, es decir por obstrucción, o problemas del sistema de alimentación o si agarro un auto electrónico puede ser el sensor del flujo de aire, porque si el sensor me está diciendo que me está mandando 20 de aire y en realidad me está mandando 10 , el aumento total de combustible hace que la mezcla me genere el humo negro..." (Héctor).

El mecánico puede reflexionar desde la práctica mientras inmerso en ella, puede hacer emerger y criticar su comprensión del fenómeno construyendo una nueva descripción de él mediante un experimento de encuadre. Cuando reflexiona desde la acción no es dependiente de las categorías de la teoría y la técnica establecida, sino que construye una nueva teoría de un caso único. Puede ser que el oxígeno que entre no sea suficiente (la mezcla es rica en mucho combustible) o que la bomba inyectora está enviando 
mucho combustible, esto dependerá del sistema de control de aire o del sistema de alimentación. El resultado es el mismo (humo negro), pero la causa es distinta.

Siguiendo la lógica de lo más sencillo a lo más complejo, comienza por pensar en el filtro de aire obstruido (esto impide la entrada adecuada de oxígeno). De no haber un filtro de aire obstruido, piensa en la temperatura del aire

“...Porque la computadora, siempre considerando un auto con inyección electrónica, controla la cantidad, el flujo del aire y la temperatura del auto, entonces todo esto va modificando el combustible...".

Si no es la temperatura del aire, piensa en la temperatura del motor, y en caso que no sea ninguna de estas cuestiones, descarta que la causa del humo negro esté relacionada con el sistema de control de aire. Entonces se concentra en el sistema de alimentación integrado por la bomba inyectora, ya que es allí donde se produce la mezcla de oxígeno y combustible. Si la mezcla está bien, piensa en los sensores.

"Yo tengo que conocer los sistemas, el sistema de alimentación el sistema electrónico, el sistema convencional. En el electrónico además tenés la computadora que es un problema también, tengo que agregar el sensor de aire, que en los sistemas comunes no los tenías..." (Héctor).

El desarrollo de competencias de diagnóstico de los mecánicos, se vincula con el enfrentarse reiteradamente a procesos reflexivos en la búsqueda de fallas electrónicas. En este contexto, los mecánicos mediante su "transacción" con la situación-problema y la reflexión que esto implica, logran resolver las distintas situaciones de fallas, desarrollando capacidades cada vez más "efectivas" para identificar fallas en los sistemas electrónicos en el automóvil. Se trata de un proceso de indagación, a través del cual el operador "gestiona" la tensión entre encuadrar el problema y mantener abiertas las líneas de indagación, a través de un proceso de interacción constante con los materiales de la situación de falla. De esta manera tiene lugar el proceso de apropiación de los nuevos artefactos de diagnóstico y de integración de las distintas bases tecnológicas.

En la medida en que su práctica se hace estable, aporta los mismos casos y se hace menos objeto de la sorpresa. Su saber desde la práctica tiende a hacerse cada vez más tácito, espontáneo y automático, pero puede perder oportunidades de pensar lo que está haciendo. El mecánico, desde su acción, a través de la reflexión, puede hacer emerger y criticar las comprensiones tácitas que maduraron en torno a las experiencias repetitivas de una práctica especializada, y puede dar un nuevo sentido a las situaciones de incertidumbre.

71 En el marco del "enfoque transaccional" desarrollado por los mecánicos -como la forma en que pueden apropiarse del instrumental de diagnóstico-, y mediante las "mediaciones epistémicas" y "pragmáticas" antes mencionadas, los mecánicos van construyendo una guía tácita de indagación de fallas -no formalizada en ningún manual-, que les marca una secuencia de descarte e intervención sobre los distintos sistemas y componentes. La presencia de esta guía tácita, elaborada a partir de la práctica cotidiana, también señala la diferencia entre el mecánico experto, -formado y socializado en la tecnología mecánica y que atravesó el proceso de "reconversión cognitiva" que implicó la introducción de la electrónica-, respecto del ayudante-novato, recién incorporado al sector. En este sentido, uno de los mecánicos señala que : 
“...Ahora es más integrado todo... ojo, yo siempre tuve la idea siempre de la integración, yo fui a un curso de esos justamente, porque en esa época... antiguamente había un especialista en motores, uno en caja, uno en frenos, uno en electricidad y otro en electrónica, yo ya estudié en integración, la idea es esa de que un tipo haga todo, mecánica, electrónica...”. (Juan)

"Fui aprendiendo con la práctica..." ; “...Estaban los manuales pero tenia una base fuerte que era el taller...”“...todo es con práctica... lo que yo aprendí de diagnóstico fue con la práctica, yo no lo aprendí porque soy inteligente...lo aprendí porque cuantas veces la gente va (otros mecánicos), lo pone (conecta el scanner), enseñame, practicás, y ahí lo importante es aprender el método, como en todos los sistemas. Si vos no conocés el sistema electrónico pero conocés el sistema de alimentación, de combustión, terminás conociendo el sistema electrónico, poco es la diferencia". "Vos tenés que saber qué función cumple cada sistema, cómo funciona y cada paso hasta darte cuenta por donde viene la falla...". (Juan)

\section{Conclusión}

72 A lo largo del artículo se ha intentado describir cómo los operadores desarrollan sus competencias de diagnóstico de fallas en los sistemas electrónicos del automóvil, a partir de la introducción de nuevas tecnologías de ayuda al mismo.

El enfoque de la reflexión desde la acción brinda elementos para discutir con enfoques que piensan a la innovación exclusivamente desde la racionalidad instrumental, en forma lineal, y con las posturas cientificistas del management. El debate entre lo que se denomina la "Racionalidad Técnica" o la "Epistemología positivista de la práctica“, y el de la "Reflexión desde la Acción" (Schön, op. cit.) -postura adoptada en este trabajopermite caracterizar la apropiación instrumental y el desarrollo de competencias de diagnóstico de fallas en la mecánica del automóvil, al mismo tiempo que permite comprender mejor de qué forma los operadores de los talleres independientes compensan los desajustes en los procesos de transferencias tecnológicas. El enfoque de la racionalidad técnica, parte de concebir la resolución de problemas instrumentales a través de la aplicación "lineal" de la teoría científica y la técnica, desde un ajuste instrumental medios-fines. La ciencia básica produce ciencia aplicada que estructura y define la práctica profesional en la resolución de problemas en contextos específicos. Por el contrario, el enfoque desarrollado por distintos segmentos de los mecánicos centrados en la "experiencia del taller", se acerca a la perspectiva de la "reflexión desde la acción" donde los problemas no se presentan como dados, sino que deben ser construidos a partir de los "materiales" de las situaciones de trabajo. Buscan poder dar sentido a la situación, imponiendo coherencia a la misma, identificando en que dirección modificarla. Desde este enfoque, al construir el problema, el mecánico puede "eludir" las categorías de la ciencia aplicada donde los fines y los medios son difusos y conflictivos, desarrollando múltiples perspectivas que estructuran su acción.

A partir del análisis de estos casos se pudo poner en evidencia un proceso en el cual los mecánicos integran conocimientos de distintas bases tecnológicas, mediante el desarrollo de una perspectiva sistémica a través de "traducciones", "decodificaciones" y "recodificaciones” entre la mecánica, la electrónica, la electricidad y la informática. Perspectiva sistémica cuyo desarrollo está vinculado al incremento de las relaciones 
funcionales entre los componentes del automóvil, a partir de la incorporación de los dispositivos electrónicos.

Los operadores desarrollan un "esquema transaccional" con los "materiales" de cada situación problemática. Al enmarcar la situación -a través de su enfoque sistémico y de su repertorio-, en paralelo mantienen abiertas las distintas líneas de indagación. Asimismo, mediante "mediaciones epistémicas" y "pragmáticas" con sus objetos de trabajo, los mecánicos construyen una guía tácita de indagación de fallas, que les señala la secuencia de descarte de fallas posibles y las líneas de intervención más pertinentes sobre los distintos sistemas y componentes.

Ahora bien, estos elementos otorgan "ventajas" a los mecánicos "expertos" para los procesos de diagnosis, respecto de los ayudantes, recién incorporados al servicio (Rouse, 1979). Los primeros han desarrollado una guía tácita más compleja y sofisticada que les permite contar con mayores recursos "cognitivos" para abordar la diversidad de situaciones de falla que se le pueden presentar.

77 Como conclusión más general, podemos sostener que, en un plano cultural, respecto de la apropiación de los objetos técnicos de ayuda al diagnóstico de fallas, los resultados parecen indicar que las limitaciones impuestas por las terminales automotrices en cuanto a las transferencias de tecnologías a los talleres independientes, son sorteadas exitosamente en los casos estudiados a partir de la apropiación que realizan los operadores mediante las estrategias de experimentación sobre los casos-problema a los cuales se ven confrontados. En los talleres independientes este proceso es más lento, basado más en estrategias de ensayo y error, requiriendo de mayores instancias de experimentación. En este sentido, quizás sería pertinente realizar estudios comparativos que permitan cotejar cómo se produce la apropiación de las innovaciones en los talleres oficiales dado que a priori, estas estrategias se verían reducidas ya que los equipos se ajustan a lo requerido por cada marca y modelo de vehículo.

78 Dichas estrategias, en un plano global, les permite a los operadores de los talleres independientes estudiados transformar una transferencia tecnológica "desajustada" en una "exitosa", a pesar de las restricciones impuestas en términos de acceso a la información, desarrollando un camino alternativo "exitoso" para la apropiación de estos artefactos superando así los desajustes "informativos".

Este tipo de realidad, inexistente en la época en la cual Alain Wisner comenzó a desarrollar su antropotecnología, impone nuevos desafíos a la disciplina y nuevas categorías para pensar estos procesos espontáneos, de adaptación, que se producen en los países que Wisner denominaba en vías de desarrollo industrial, y que hoy se los llama emergentes.

80 Complementariamente, en el plano de la actividad instrumental, la puesta a disposición en el mercado de "artefactos genéricos" de ayuda al diagnóstico de fallas parece operar en forma positiva respecto de los procesos de apropiación de estas tecnológicas, por parte de los operadores de talleres independientes. Dichos artefactos, les brindan la posibilidad de generar verdaderas génesis instrumentales en sus procesos de diagnóstico y resolución de fallas, asegurando de esa forma la adaptación al sistema productivo.

81 Asimismo, los resultados de esta indagación parecen mostrar que las actividades de experimentación y de reflexión en la acción desarrolladas por los operadores, les permiten generar unos conocimientos basados en los casos abordados mostrando de 
esta forma que, si bien los manuales resultan en primera instancia necesarios, son insuficientes si se considera que la actividad de diagnóstico no se reduce a una actividad de mera asociación entre los casos y la nomenclatura de fallas posibles. No se trata, de ningún modo, de la mera aplicación de una regla sino que, muchas veces, tal como los muestran los casos abordados se trata más bien de su construcción.

Por otra parte, el estudio muestra en el plano de la cognición, hasta qué punto la introducción de la electrónica y la informática en el automóvil modifica el contenido de las tareas en la medida que, por un lado los diferentes subsistemas son más interdependientes $\mathrm{y}$, por otro lado, introducen un alejamiento del objeto de trabajo, tornando la actividad mental más compleja, más abstracta.

Finalmente, es de considerar que si bien en estos últimos treinta años muchos son los cambios que han transformado sensiblemente al mundo del trabajo, tampoco es menos cierto que los procesos de transferencia de tecnologías no han cesado de multiplicarse e, incluso, de acelerarse y de tomar nuevas formas. Por citar otros ejemplos, que no se han abordado en este trabajo, se han transferido y se han incorporado innovaciones tecnológicas a los procesos de producción de bienes y servicios en los países emergentes, como ser las nanotecnologías y los organismos genéticamente modificados en agricultura, cuyos riesgos merecen ser estudiados en profundidad, cuenta habida del impacto social que dichas innovaciones conllevan, en términos de salud y seguridad de las poblaciones. En este sentido el programa planteado por la antropotecnología pareciera destinado a reformularse para considerar y dar respuesta a estos nuevos desafíos.

\section{BIBLIOGRAFÍA}

Delfini, M., Dubbini, D., \& Rivero, I. (2007). (Compiladores), Articulación y desarrollo de competencias en la trama automotriz argentina : morfología, innovación y empleo en Innovación y empleo en tramas productivas argentinas. Buenos Aires : Prometeo libros.

Folcher, V., \& Rabardel, P. (2009). Hombres, artefactos, actividades : perspectiva instrumental. In P. Falzon (Ed.), Ergonomie (pp. 247-279). Paris : PUF. Edición Española ; Madrid : Modus Laborandi.

Geslin, Ph. (2004). Agir de concert. Anthropotechnologie et recherches anthropologiques. In J. Durafour, \& B. Vuillon, Alain Wisner et les tâches du présent (pp. 75-83). Toulouse : Octarès Editions.

Granovsky, P (2010). Innovación y cambio tecnológico : análisis del impacto en las calificaciones de los trabajadores mecánicos a partir de la introducción de la electrónica en el automóvil. Tesis de maestría. Facultad de Ciencias Sociales. Universidad de Buenos Aires, Buenos Aires.

Guèrin, F., Laville, A., Daniellou, F., Duraffourg, J., \& Kerguelen, A. (2006). Comprendre le travail pour le transformer. La pratique de l'ergonomie. Lyon : Éditions du réseau ANACT.

Leplat, J. (1997). Regards sur l'activité en situation de travail. Paris : Editions PUF.

Leplat, J. (2000). L'analyse psychologique de l'activité en ergonomie. Aperçu de son évolution, ses modèles et ses méthodes. Toulouse : Octarès Editions. 
Nonaka, I., \& TakeuchI, H. (2002). La organización creadora de conocimiento. Cómo las compañías japonesas crean la dinámica de la innovación. México : Oxford.

Rabardel, P. (1995). Les hommes et les technologies. Approche cognitive des instruments contemporains. Paris : Armand Collin.

Rogalsky, J., \& Marquié, J. C. (2004). Évolutions des compétences et des performances. In J.-M. Hoc, \& F. Darses, Psychologie ergonomique. Tendances actuelles (pp. 141-173). Collection Le Travail Humain. Paris : Editions PUF.

Rouse, W. B. (1979). Problem solving performance of first semester maintenance trainees in two fault diagnosis tasks. Human factors, 21(5), 611-618.

Rouse, W.B., \& Rouse, S.H. (1979). Measures of complexity fault diagnostic tasks. IEEE Transactions on systems, Man, and cybernetics, SMC-9, 720-727.

Schon, D. (1998). El profesional reflexivo. Cómo piensan los profesionales cuando actúan. Barcelona : Editorial Paidós.

Wisner, A. (1981). Vers une antropotechnologie, Comment pourvoir les pays en développement industriel de machines et d'usines qui marchent. Rapport de recherche. Paris : Laboratoire de Physiologie du Travail et d'Ergonomie, Conservatoire National des Arts et Métiers.

Wisner, A. (1985). Quand voyagent les usines. Collection Atelier futur. Paris : Editions Syros.

\section{NOTAS}

1. Se trata de un Programa multisectorial de certificación de competencias laborales, financiado por el Fondo Multilateral de Inversiones del Banco Interamericano de Desarrollo, ejecutado durante el período 2003-2009.

\section{RESÚMENES}

Los procesos de innovación tecnológica implican grandes desafíos para las diferentes disciplinas interesadas por el trabajo. En un plano cultural, por la diversidad de los contextos socioculturales donde esas tecnologías se transfieren; en el plano cognitivo, por las modificaciones que las innovaciones implican en el contenido de las tareas, en la apropiación de los objetos técnicos, y en cuanto a la modificación o construcción de esquemas de utilización de los artefactos.

Este estudio se refiere al impacto producido por la introducción de la electrónica en los componentes del automóvil y a sus consecuencias sobre el trabajo de diagnóstico de fallas, en los operadores formados en la "mecánica tradicional", haciendo foco en los procesos de apropiación de nuevas técnicas de diagnóstico de fallas en los automóviles. En particular, este trabajo se centra sobre la actividad de los operadores de mantenimiento y reparación de automóviles en talleres independientes, que están por fuera del circuito de las concesionarias oficiales y que, por lo tanto, no reciben de los fabricantes los dispositivos técnicos necesarios (scanner, osciloscopio y manuales de diagnóstico).

Los datos relevados muestran que el razonamiento de los operadores resulta una invariante que 
les permite apropiarse de las modificaciones que introducen las innovaciones tecnológicas en las tareas de diagnóstico de fallas, permitiendo compensar los déficits que revelan "una transferencia tecnológica desajustada".

Os processos de inovação tecnológica colocam um desafio importante às disciplinas que se interessam pelo trabalho: tendo em conta o impacto desses processos sobre um plano cultural, considerando a diversidade dos contextos sócio-culturais em que estas tecnologias são transferidas; e sobre um plano cognitivo, pelas modificações das tarefas e mecanismos de apropriação dos objectos técnicos que eles induzem, assim como pelas modificações e construções de novos esquemas de utilização dos artefactos.

Este estudo analisa o impacto da introdução da eletrónica nos componentes automóveis sobre os operadores formados em "mecânica tradicional", salientando os processos de apropriação de novas técnicas de diagnóstico de avarias nestes veículos. O trabalho de pesquisa visa particularmente a atividade dos operadores de manutenção e de reparação de automóveis em oficinas independentes, que estão fora do circuito dos concessionários oficiais e que, por isso, não recebem dos fabricantes os dispositivos técnicos necessários (scanner, osciloscópio, e manuais de apoio ao diagnóstico de avarias).

Os dados colocam em evidência que o raciocínio sistémico dos operadores constitui uma invariante que lhes permite apropriarem-se das modificações que são introduzidas pelas inovações tecnológicas neste contexto particular da tarefa de diagnóstico de avarias, permitindo assim compensar os défices que decorrem de "uma transferência tecnológica desajustada".

Les processus d'innovation technologique posent un défi important aux disciplines intéressées par le travail : compte tenu de l'impact de ces processus sur un plan culturel, si on considère la diversité des contextes socio-culturels où les technologies se transfèrent; et sur un plan cognitif, suite aux modifications des tâches et mécanismes d'appropriation des objets techniques qu'ils induisent, ainsi qu'en raison de la modification et construction de nouveaux schèmes $\mathrm{d}$ 'utilisation des artefacts.

Cette étude se centre sur l'impact produit par l'introduction de l'électronique dans les composants de l'automobile sur les opérateurs formés à la "mécanique traditionnelle", en mettant l'accent sur les processus d'appropriation des nouvelles techniques de diagnostic de pannes d'automobile. En particulier, cette enquête vise l'activité des opérateurs de maintenance et de réparation de voitures dans des garages indépendants, qui ne sont pas équipés par les constructeurs de dispositifs techniques nécessaires (scanner, oscilloscope, et des manuels d'aide au diagnostic de pannes).

Les données relevées mettent en évidence que le raisonnement systémique des opérateurs $\mathrm{s}$ 'avère être un invariant qui leur permet de s'approprier des modifications, issues des innovations technologiques, dans ce contexte particulier de la tâche de diagnostic de pannes, permettant ainsi de compenser les déficits qui découlent d'un "transfert technologique déréglé".

The processes of technological innovation involve big challenges for the different disciplines interested in work issues, taking into account the impacts that the above mentioned processes carry, among others: on a cultural level, in terms of the diversity of the socio-cultural contexts where these technologies are transferred. From a cognitive point of view, such innovations involve changes in the content of the tasks, in the mechanisms of appropriation of technical objects, and in the modification of schemes of utilization of the appliances.

This study specifically addresses the impact produced by the introduction of electronics in the components of the car, on the work of operators trained in "traditional mechanics ", focusing on the processes of appropriation of new techniques for diagnosing faults in vehicles. In particular, this paper focuses on the activity of maintenance operators and independent car repair workshops, organizations that are outside the official circuit of car dealerships and therefore not 
dependent on the manufacturers for the provision of technical devices (scanners, oscilloscopes and manuals).

The information collected shows that the previous systemic reasoning, of the operators, turns out to be an invariant that allows them to appropriate the changes introduced by technological innovations in fault diagnosis tasks, in the task of diagnosis of faults allowing to compensate the deficits that reveal "a technological poorly adjusted transfer".

\section{ÍNDICE}

Palabras claves: transferencia de tecnologías, apropiación instrumental, competencias, actividad de diagnóstico

Mots-clés: transfert de technologies, appropriation instrumentale, compétences, activité de diagnostic

Keywords: technological transfer, instrumental appropriation, skills, diagnosis activity

Palavras-chave: transferência de tecnologia, apropriação instrumental, competências, atividade de diagnóstico

\section{AUTORES}

\section{PABLO GRANOVSKY}

Universidad Nacional de la Matanza, San Justo - Buenos Aires, Florencio Varela 1903, (B1754JEC) San Justo, Pcia. de Bs. As. - Argentina

pgranovsky2004@yahoo.com.ar

\section{MÁRIO POY}

Centro de Investigaciones por una Cultura de Seguridad, Universidad de San Andrés -

Universidad de Palermo, Victoria - Buenos Aires, Vito Dumas 284,(B1644BID) Victoria, Pcia. de Bs. As.- Argentina mpoy@udesa.edu.ar 